\title{
A survey of obstetric complications and pregnancy outcomes in paediatric and nonpaediatric anaesthesiologists
}

VIRGINIA T. GAUGER MD, TERRI VOEPEL-LEWIS MSN, RN, PHILIP RUBIN BS, AMY KOSTRZEWA MD AND

ALAN R. TAIT PhD

University of Michigan, Ann Arbor, MI, USA

\section{Summary}

Background: Obstetric complications such as spontaneous abortion, preterm labour, preterm delivery, low birth weight and congenital anomalies may be associated with exposure to anaesthetic gases. We hypothesized that female anaesthesiologists practicing primarily paediatric anaesthesia, with increased exposure to trace anaesthetic agents, experience a greater prevalence of obstetric complications than female anaesthesiologists performing primarily adult anaesthesia. Methods: Questionnaires were sent to all female Society for Pediatric Anesthesia (SPA) members and to an equal number of randomly selected female American Society of Anesthesiologists (ASA) members. Subjects were asked to answer questions regarding their pregnancy outcomes, work history and personal habits. Parametric data were analysed by unpaired $t$-tests. Nonparametric data were analysed by chi-square, Fisher's exact test and Mann-Whitney $U$-test as appropriate.

Results: Paediatric anaesthesiologists were defined as those having $>75 \%$ paediatric practice. Paediatric anaesthesiologists were older and had greater operating room exposure during their pregnancies than nonpaediatric anaesthesiologists. There was a significantly higher prevalence of spontaneous abortion among paediatric anaesthesiologists than nonpaediatric anaesthesiologists. In an exploratory analysis, the following factors were found to be significantly associated with the development of spontaneous abortion: age $>35$ years, gravida $>1$, exercise during pregnancy, percentage of inhalational anaesthetics $>75 \%$ and paediatric anaesthesia practice $>75 \%$. Independent risk factors for spontaneous abortion among anaesthesiologists included exercise ( $>1$ time/week) and age.

Conclusion: Our results suggest a higher prevalence of spontaneous abortion in anaesthesiologists whose practice is $>75 \%$ paediatrics.

Keywords: paediatric anaesthesia; trace anaesthetic gases; spontaneous abortion 


\section{Introduction}

Obstetric complications such as spontaneous abortion, preterm labour, preterm delivery, low birth weight and congenital anomalies have been associated with exposure to anaesthetic gases. Although diethyl ether was first introduced in the 1840s, it was not until 1967 that the first study addressing the adverse reproductive effects of inhalational agents was published. In this paper from the Soviet Union, Vaisman reported that 18 of 31 pregnancies among anaesthesiologists exposed to waste anaesthetic gases ended in spontaneous abortion (1). Since that time, numerous epidemiological studies have been performed, the most notable and influential being the 1974 national study supported by the American Society of Anesthesiologists (ASA) which suggested that female operating room personnel were at increased risk for spontaneous abortion and that their children had a greater prevalence of congenital anomalies (2).

Concerns were great enough that in 1977, the National Institute for Occupational Safety and Health (NIOSH) established standards for acceptable maximum levels of environmental trace anaesthetic gases. It was thought that careful anaesthetic technique, room ventilation and implementation of scavenging systems would decrease pollution to acceptable levels. Despite the importance of these guidelines, paediatric anaesthesia presents some unique problems of waste spillage because of the use of mask inductions and uncuffed tracheal tubes. In fact, several recent publications have supported this concept. In 1992, Wood et al. found that exposures of the anaesthetist, nurse and surgeon to nitrous oxide during routine paediatric otolaryngeal surgery exceeded the NIOSH recommendations regardless of the induction technique used (3). Furthermore, Hoerauf et al. showed levels in excess of the United Kingdom Committee for Occupational Safety and Health's (COSH) recommendations for the anaesthetist and circulating nurse during mask induction of paediatric patients for ophthalmic surgery (4).

Based on this observation, we hypothesized that female anaesthesiologists practicing primarily paediatric anaesthesia, with increased exposure to trace anaesthetic agents, would experience a greater prevalence of obstetric complications compared with female anaesthesiologists performing primarily adult anaesthesia.

\section{Methods}

The study was approved by The University of Michigan Medical School Institutional Review Board for Human Subject Research.

In developing the questionnaire, a list of constructs was generated to define the categories of interest. These constructs were derived from a review of the literature and expert opinion. Following the generation of these constructs, several of our anaesthesiology colleagues were interviewed in order to generate a list of potential items (questions) that would describe the constructs of interest. These items formed the basis of a pilot questionnaire which was tested for face and content validity when administered to a sample of both anaesthesiologists and other nonphysician personnel. In addition to general demographic questions, the questionnaire contained items addressing pregnancy outcomes, work history, and personal habits such as exercise, smoking, alcohol consumption and illicit drug use. Furthermore, a global assessment of the anaesthesiologists' perceived overall stress during pregnancy was obtained using a $0-10$ visual analogue scale where $0=$ no stress and $10=$ most stress possible.

Following pilot testing, a final questionnaire was developed which was sent to all female members of the Society for Pediatric Anesthesia (SPA) and an equal number of female members of the ASA randomly selected using systemic sampling with a random start. The questionnaire was sent with an accompanying letter explaining the purpose of the study and a self-addressed, postage-paid envelope for return of the completed questionnaire. In an attempt to reduce the potential for self-report bias, the questionnaire was anonymous and contained no identifying information. Because of the anonymous nature of the questionnaire, the subjects were asked to return a prepaid postcard separately from the questionnaire indicating that it had been completed and returned. In this manner, subjects could be identified as respondents without observer knowledge of which questionnaire was theirs. If there was no response to the questionnaire after 4 weeks, a second mailing was sent out to all nonrespondents. 
Statistical analysis was performed using SPSS software (SOPSS Inc., Chicago, IL, USA). Sample size determination was based on the anticipated number of eligible female anaesthesiologists obtained from the SPA and a matching number from the ASA. Frequency distributions were computed for descriptive data. Parametric data were analysed by unpaired $t$-tests. Nonparametric data were analysed by chi-square, Fisher's exact test and Mann-Whitney $U$-test as appropriate. Significance was accepted as $P<0.05$.

\section{Results}

A total of 1948 surveys were mailed, of which 824 were returned, indicating a response rate of $42 \%$. For the purpose of this study, a multiple gestation was considered as a single pregnancy. Forty-four of the pregnancies were unable to be allocated to paediatric practice vs nonpaediatric practice categories and 11 of the pregnancies had no data on obstetric outcomes. Consequently, analysis was performed on 1506 pregnancies. Paediatric anaesthesiologists were defined as those having $>75 \%$ paediatric practice. The demographics of the study sample are described in Table 1 . There were no

Table 1

Demographics $[n(\%)]$

\begin{tabular}{lcc}
\hline & $\begin{array}{c}\text { Nonpaediatric } \\
\text { anaesthesiologists } \\
(\mathrm{n}=1275)\end{array}$ & $\begin{array}{c}\text { Paediatric } \\
\text { anaesthesiologists } \\
(\mathrm{n}=231)\end{array}$ \\
\hline Age (years, mean $\pm \mathrm{SD})$ & $33.28 \pm 4.03^{*}$ & $34.59 \pm 3.27$ \\
Race & $738(78.8)$ & $139(84.8)$ \\
$\quad$ Caucasian & $26(2.8)$ & $5(3.0)$ \\
$\quad$ African-American & $3(0.3)$ & $3(1.8)$ \\
Hispanic & $170(18.1)$ & $17(10.4)$ \\
Other & $2 / 1$ & $2 / 1$ \\
Gravida/Primipara (median) & $1186(97)$ & $208(96)$ \\
Prenatal care & $25(2)$ & $7(3)$ \\
Tobacco use (>1 cigarette/day) & $202(16)$ & $33(14.3)$ \\
Alcohol use $(>1$ drink/week) & $6(0.5)$ & $1(0.5)$ \\
Illicit drug use & $705(57.1)$ & $118(52.4)$ \\
Exercise ( $>1$ time/week) & $6.22 \pm 2.33$ & $6.18 \pm 2.35$ \\
Overall stress (mean \pm sD) $\dagger$ & $890(70.6)^{*}$ & $183(79.2)$ \\
OR exposure $(>40 h /$ wk) & $585(47)$ & $98(43.4)$ \\
Night shift $(>4 /$ month) & $1045(88)$ & $201(93.9)$ \\
OR scavenging systems & $741(63.6)$ & $143(66.8)$ \\
OR ventilation/air exchange & $946(77.5)$ & $162(72.6)$ \\
Worked through & & \\
third Trimester & & \\
\hline
\end{tabular}

${ }^{*} P<0.007$ vs paediatric anaesthesiologists. $+0-10$ scale where $10=$ extremely stressed, $\mathrm{OR}=$ operating room. differences between paediatric and nonpaediatric anaesthesiologists with respect to race/ethnicity, para/gravida status, prenatal care, or social habits (tobacco, alcohol, illicit drug use). Paediatric anaesthesiologists were statistically older (1.3 years) than nonpaediatric anaesthesiologists. Despite this, there were no differences in the prevalence of spontaneous abortion between paediatric anaesthesiologists $>35$ years of age $(20 \%)$ and those $<35$ years of age $(22.3 \%)$. Paediatric anaesthesiologists also had greater operating room (OR) exposure during their pregnancies than nonpaediatric anaesthesiologists. However, there was not a significant difference in the prevalence of spontaneous abortion in paediatric anaesthesiologists who had $>40 \mathrm{~h} /$ week of OR exposure as compared with those with $<40 \mathrm{~h} /$ week $(22.4 \%$ vs $16.7 \%)$. There were no differences between groups with respect to the number of night shifts worked or work environment (OR scavenging, air/ventilation systems). There were also no differences between paediatric anaesthesiologists and nonpaediatric anaesthesiologists with respect to the prevalence of coexisting conditions (Table 2).

Pregnancy outcomes and the prevalence of birth defects or anomalies are described in Table 3. There were no differences between paediatric and nonpaediatric anaesthesiologists with respect to preterm labour, preterm delivery, birth weight or the appearance of birth defects. However, there was a significantly higher prevalence of spontaneous abortion among paediatric anaesthesiologists. There was also a $4 \%$ lower incidence of live-births among paediatric anaesthesiologists; however, this did not achieve statistical significance.

Table 2

Coexisting conditions among female anaesthesiologists $[n(\%)]$

\begin{tabular}{lcc}
\hline Coexisting condition & $\begin{array}{c}\text { Nonpaediatric } \\
\text { anaesthesiologists }\end{array}$ & $\begin{array}{c}\text { Paediatric } \\
\text { anaesthesiologists }\end{array}$ \\
\hline Sexually transmitted disease & $9(0.7)$ & $4(1.7)$ \\
Bacterial vaginosis & $7(0.6)$ & $1(0.4)$ \\
Urinary tract infection & $42(3.3)$ & $13(5.7)$ \\
Uterine abnormality & $20(1.6)$ & $5(2.2)$ \\
Incompetent cervix & $24(1.9)$ & $3(1.3)$ \\
Uteroplacental insufficiency & $14(1.1)$ & $2(0.9)$ \\
Uterine irritability & $106(8.4)$ & $21(9.1)$ \\
Hypertension & $52(4.1)$ & $12(5.2)$ \\
Gestational diabetes & $30(2.4)$ & $3(1.3)$ \\
Preeclampsia/eclampsia & $57(4.5)$ & $9(3.9)$ \\
\hline
\end{tabular}


Table 3

Pregnancy outcomes among female anaesthesiologists $[n(\%)]$

\begin{tabular}{lcc}
\hline Outcome & $\begin{array}{c}\text { Nonpaediatric } \\
\text { anaesthesiologists }\end{array}$ & $\begin{array}{c}\text { Paediatric } \\
\text { anaesthesiologists }\end{array}$ \\
\hline Spontaneous abortion & $194(15.2)^{*}$ & $49(21.2)$ \\
Live-births & $1047(82.2)$ & $179(78.2)$ \\
Preterm labour & $154(12.2)$ & $34(14.7)$ \\
Gestational age (weeks) & $36.2 \pm 8.5$ & $35.4 \pm 9.9$ \\
Birth weight (lbs, mean \pm sD) & $7.27 \pm 1.23$ & $7.38 \pm 1.21$ \\
Birth defects/anomalies & & \\
Cardiac & $22(1.7)$ & $1(0.4)$ \\
Respiratory & $11(0.9)$ & $2(0.8)$ \\
Musculoskeletal & $26(2.0)$ & $3(1.3)$ \\
Gastrointestinal & $6(0.5)$ & $2(0.9)$ \\
$\quad$ Neurological & $17(1.3)$ & $3(1.3)$ \\
Skin & $21(1.7)$ & $2(0.9)$ \\
Other & $64(5.0)$ & $15(6.5)$ \\
\hline
\end{tabular}

${ }^{*} P<0.05$ vs paediatric anaesthesiologists.

Given the association between paediatric anaesthesia and spontaneous abortion, we performed an exploratory analysis of several other factors to determine their contribution to the development of this outcome. Variables included: age, race, gravida/ para status, prenatal care, tobacco/alcohol/illicit drug use, exercise habits, stress, hours of OR exposure, night shifts worked, ORs with scavenging and/or ventilation systems, number of trimesters worked and percentages of time performing inhalational anaesthesia. Other variables included: histories of sexually transmitted diseases, bacterial vaginosis, urinary tract infection, uterine abnormality, incompetent cervix, uteroplacental insufficiency, uterine irritability, hypertension, gestational diabetes and preeclampsia/eclampsia. Of these, several factors were found to be significantly associated with the development of spontaneous abortion. These included: age $>35$ years [(odds ratio 2.1 (95\% confidence interval, 1.6, 2.8), $P=0.0001]$, gravida status $>1[1.4(1.1,1.9), P=0.02]$, exercise during pregnancy $[1.6(1.2,2.1), P=0.002]$, percentage of inhalation anaesthetics $>75 \%[1.4(1.0,2.1)$, $P=0.049]$, and paediatric anaesthesia practice $>75 \%$ [1.5 (1.1, 2.1), $P=0.023]$. These factors and their interactions (e.g. paediatric anaesthesiologists by age) were subsequently entered into a logistic regression model with backward selection. Multivariate analysis of these factors yielded two independent risk factors for spontaneous abortion among anaesthesiologists: age $(P<0.0001)$ and exercise
$>1$ time/week $(P=0.006)$. There was no significant interaction between age and paediatric anaesthesia practice $>75 \%$.

\section{Discussion}

Our results suggest a higher prevalence of spontaneous abortion in anaesthesiologists whose practice is $>75 \%$ paediatrics. As this is the only study that has surveyed paediatric and nonpaediatric anaesthesiologists, there are no comparative data. However, the hypothesis was that female anaesthesiologists practicing primarily paediatric anaesthesia would be at increased risk of poor obstetric outcomes. The rationale for this was that paediatric anaesthesiologists have an increased exposure to trace anaesthetic gases during inhalation inductions and the use of uncuffed tracheal tubes.

Despite the fact that there are no previous studies comparing paediatric and nonpaediatric anaesthesiologists, there are numerous other epidemiological studies comparing exposed OR groups to nonexposed groups. Three early studies in the former USSR (1), Denmark (5), and the USA (6) all suggested an association between anaesthetic practice by pregnant women and an increased frequency of spontaneous abortion. These findings were later confirmed by three large surveys, two in the UK $(7,8)$ and one in the USA $(2)$. In the study by Cohen et al., supported by the ASA, some 40000 members from various professional organizations were surveyed (6). Female respondents exposed to OR trace anaesthetics were found to have a 1.3-2 time risk of spontaneous abortion as compared with unexposed women. Following this, the ASA commissioned a group of epidemiologists and biostatisticians who published the first of two meta-analyses addressing this issue. In this study that summarized six epidemiological papers, Buring et al. showed a $30 \%$ increased risk of spontaneous abortion for women working in the operating room (9). The second meta-analysis, published by Boivin in 1997, calculated a relative risk of 1.48 for spontaneous abortion in women occupationally exposed to anaesthetic gases (10). Our data suggest that the risk of spontaneous abortion for paediatric anaesthesiologists is similar to these previous reports. The meta-analyses, therefore, lend external validity to our data. 
Despite the consistency of these findings, there are epidemiological studies refuting this association. In 1977, Pharoah et al. showed that prevalence of spontaneous abortion was $13.8 \%$ for both female anaesthetists and female 'others' (medical appointment other than anaesthetist) (11). Their survey, however, provided no information regarding gas exposure prior to and during the pregnancies. In another study, Rosenberg and Vanttinen found the rate of spontaneous abortion to be $10.2 \%$ in anaesthetists and $13.2 \%$ in paediatricians (12). These numbers pooled both maternal and paternal occupational exposure. Neither of these studies mentioned whether scavenging or ventilation/air exchange systems were employed and, thus, it is possible that OR exposure to trace anaesthetic gases may have been lower than in the previously mentioned studies.

In our study, the paediatric and nonpaediatric anaesthesiologist groups differed in two demographic variables, age and hours of OR exposure. As these factors could potentially confound our outcome measures, we compared the prevalence of spontaneous abortion in paediatric anaesthesiologists who were less than and greater than 35 years of age and those who had less than and greater than $40 \mathrm{~h} /$ week of OR exposure. As reported in the 'Results', there was no statistically significant difference in the prevalence of spontaneous abortion with respect to these two variables, suggesting that the confounding effects of age and OR exposure may be minimal.

Furthermore, we found two independent risk factors of spontaneous abortion: age and exercise $>1$ time/week. Increasing maternal age has repeatedly been associated with spontaneous abortion. In a population based register linkage study, the risk for spontaneous abortion varied according to maternal age $(8.7 \%$ for 22 years of age to $84.1 \%$ for $\geq 48$ years of age) (13). The primary cause of miscarriages in older women is thought to be the result of chromosomal deletions and fatal trisomies (14). However, other factors thought to be associated with spontaneous abortion include structural abnormalities of the uterus, poorly controlled comorbidities, genital infections, caffeine, alcohol, tobacco, trace element or chemical exposure, psychological stress, physical stress and shift work (15). Although the majority of recent literature supports exercise during preg- nancy, there are studies suggesting this may impose a greater risk of spontaneous abortion. In a prospective study, Hjollund et al. found that physical strain around the time of implantation was associated with later spontaneous abortion (16). Our data found exercise to be an independent risk factor for spontaneous abortion. However, it is limited in that we are not certain as to the type or timing of the exercise performed by these women.

As in any survey, there are limitations that necessitate caution in interpreting the results. The potential biases of this survey include the reliability of the questionnaire, self-report bias, representativeness, nonresponse bias, documentation of anaesthetic exposure levels and confounding variables, and lack of verification of outcome events. The questionnaire was tested for face and content validity and also internal consistency reliability. Self-report bias can manifest as a desire on the part of the respondent to appear in a more positive light. However, the anonymous nature of the questionnaire should have reduced any urge to intentionally downplay or exaggerate information. The sample used in this study was obtained by probability and nonprobability sampling. As the SPA represented a relatively small society, a convenience (nonprobability) sample of all female members was obtained. As such, the sample should have been representative. As the ASA is a much larger society, the sample of female members was randomly selected using systematic sampling with a random start. This latter form of sampling should also have provided a representative sample. Our response rate of $42 \%$ is comparable with the response rates seen in other surveys of health care professionals (17). In this particular study, the concern is that nonresponders differ substantially from responders. The only method to eliminate this bias is to improve the overall response rate which we attempted to do by sending out two separate mailings 4 weeks apart. Unfortunately, because of the self-report nature of this survey, the biases of anaesthetic exposure levels, confounding variables and lack of verification are difficult, if not impossible, to eliminate. In previous epidemiological studies involving trace anaesthetic gas exposure, the control groups were different from the study groups in multiple respects. We minimized these differences by using professional women in both 
groups that would have similar day schedules, job requirements and stresses.

In their recent 1999 publication on waste anaesthetic gases, the ASA concluded: 'There are no data to suggest that waste anesthetic gases are a danger to those women working in a scavenged environment who are contemplating pregnancy or who are already pregnant' (18). Our data, however, suggest that because of the unique aspects of anaesthesia management in children, female anaesthesiologists whose practice involves primarily paediatric anaesthesia may be at risk of adverse obstetric outcomes. Data from the Physician Characteristics and Distribution, 1999 Edition show a continued increase in the number of United States physicians, especially female physicians. In 1997, there were 33730 anaesthesiologists, nearly $20 \%$ (or 6707 ) of whom were female (19). If this trend continues, there will be increasingly more women exposed to anaesthetic gases. Consequently, it is important to determine whether those women choosing to practice paediatric anaesthesia are at increased risk for obstetric complications.

\section{References}

1 Vaisman AI. Working conditions in the operating room and their effect on the health of anesthesiologists. Eksperimentalnaia Khirurgiia i Anestheziologiia 1967; 12: 44-49.

2 American Society of Anesthesiologists Ad Hoc Committee on the Effect of Trace Anesthetics on the Health of Operating Room Personnel. Occupational disease among operating room personnel: a national study. Anesthesiology 1974; 41: 321-340.

3 Wood C, Ewen A, Goresky G et al. Exposure of operating room personnel to nitrous oxide during paediatric anaesthesia. Can J Anaesth 1992; 39: 682-686.

4 Hoerauf K, Funk W, Harth M et al. Occupational exposure to sevoflurane, halothane and nitrous oxide during paediatric anaesthesia. Waste gas exposure during paediatric anaesthesia. Anaesthesia 1997; 52: 215-219.

5 Askrog V, Harvald B. Teratogen effekt af inhalations anaestetika. Nordisk Medicin 1970; 83: 498-500.
6 Cohen EN, Bellville JW, Brown BW, Jr. Anesthesia, pregnancy, and miscarriage: a study of operating room nurses and anesthetists. Anesthesiology 1971; 34: 343-347.

7 Knill-Jones RP, Rodrigues LV, Moir DD et al. Anaesthetic practice and pregnancy: controlled survey of women anaesthetists in the United Kingdom. Lancet 1972; 1: 1326-1328.

8 Knill-Jones RP, Newman BJ, Spence AA. Anaesthetic practice and pregnancy: controlled survey of male anaesthetists in the United Kingdom. Lancet 1975; 2: 807-809.

9 Buring JE, Hennekens CH, Mayrent SL et al. Health experiences of operating room personnel. Anesthesiology 1985; 62: 325-330.

10 Boivin JF. Risk of spontaneous abortion in women occupationally exposed to anaesthetic gases: a meta-analysis. Occup Environ Med 1997; 54: 541-548.

11 Pharoah PO, Alberman E, Doyle P et al. Outcome of pregnancy among women in anaesthetic practice. Lancet 1977; 1: 34-36.

12 Rosenberg PH, Vanttinen H. Occupational hazards to reproduction and health in anaesthetists and paediatricians. Acta Anaesthesiol Scand 1978; 22: 202-207.

13 Nybo Andersen AM, Wohlfahrt J, Christens P et al. Maternal age and fetal loss: population based register linkage study. British Med J 2000; 320: 1708-1712.

14 Volarcik K, Sheean L, Goldfarb J et al. The meiotic competence of in-vitro matured human oocytes is influenced by donor age: evidence that folliculogenesis is compromised in the reproductively aged ovary. Human Reprod 1998; 13: 154-160.

15 Cramer DW, Wise LA. The epidemiology of recurrent pregnancy loss. Semin Reprod Med 2000; 18: 331-339.

16 Hjollund NH, Jensen TK, Bonde JP et al. Spontaneous abortion and physical strain around implantation: a follow-up study of first-pregnancy planners. Epidemiology 2000; 11: 18-23.

17 Tait AR, Tuttle DB. Prevention of occupational transmission of human immunodeficiency virus and hepatitis $B$ virus among anesthesiologists: a survey of anesthesiology practice. Anesth Analg 1994; 79: 623-628.

18 ASA Task Force on Trace Anesthetic Gases of the ASA Committee on Occupational Health of Operating Room Personnel. Waste Anesthetic Gases. Information for Management in Anesthetizing Areas and the Postanesthesia Care Unit (PACU). American Society of Anesthesiologists, 1999.

19 Physician Characteristics and Distribution, 1999 Edition, Department of Data Survey and Planning, Division of survey and Data Resources, American Medical Association, 1998.

Accepted 23 September 2002 